\title{
MRI Correlates of Parkinson's Disease Progression: A Voxel Based Morphometry Study
}

\author{
Valentina Fioravanti, Francesca Benuzzi, Luca Codeluppi, Sara Contardi, \\ Francesco Cavallieri, Paolo Nichelli, and Franco Valzania
}

Department of Neuroscience, Nuovo Ospedale Civile S. Agostino-Estense, University of Modena and Reggio Emilia, Viale Giardini 1355, 41126 Modena, Italy

Correspondence should be addressed to Valentina Fioravanti; valentina.fioravanti@gmail.com

Received 23 September 2014; Revised 23 December 2014; Accepted 23 December 2014

Academic Editor: Heinz Reichmann

Copyright ( 2015 Valentina Fioravanti et al. This is an open access article distributed under the Creative Commons Attribution License, which permits unrestricted use, distribution, and reproduction in any medium, provided the original work is properly cited.

\begin{abstract}
We investigated structural brain differences between a group of early-mild PD patients at different phases of the disease and healthy subjects using voxel-based morphometry (VBM). 20 mild PD patients compared to 15 healthy at baseline and after 2 years of follow-up. VBM is a fully automated technique, which allows the identification of regional differences in the gray matter enabling an objective analysis of the whole brain between groups of subjects. With respect to controls, PD patients exhibited decreased GM volumes in right putamen and right parietal cortex. After 2 years of disease, the same patients confirmed GM loss in the putamen and parietal cortex; a significant difference was also observed in the area of pedunculopontine nucleus (PPN) and in the mesencephalic locomotor region (MLR). PD is associated with brain morphological changes in cortical and subcortical structures. The first regions to be affected in PD seem to be the parietal cortex and the putamen. A third structure that undergoes atrophy is the part of the inferior-posterior midbrain, attributable to the PPN and MLR. Our findings provide new insight into the brain involvement in PD and could contribute to a better understanding of the sequence of events occurring in these patients.
\end{abstract}

\section{Introduction}

Neuropathological studies in Parkinson's disease (PD) have proposed a six-phase progression system, indicating a growing and predictable sequence of brain pathology related to neurological deficits presented by patients at different stages of the disease [1]. However this classification often does not timely correlate with clinical severity or with onset of specific aspects of the symptomatology, such as postural or cognitive symptoms [2].

Only few studies investigated PD patients with shortdisease duration. These studies reported no changes [3], lateral and medial frontal atrophy $[4,5]$, hippocampal/amygdala atrophy [4, 6, 7], and left cerebellum atrophy [8]. The purpose of this study was to identify cortical and subcortical structural changes at an early stage of PD and to evaluate their progression over 2 years.

The longitudinal evaluation of brain atrophy in patients with PD has been studied only in few studies with contradictory results. In fact, while Ramírez-Ruiz and collaborators showed the presence of a reduction of gray matter in several cortical areas [9], Brenneis and collaborators comparing a group of patients suffering from PD and a group of patients with multisystem atrophy (MSA-P) found a progression of the atrophy only in the group of patients with MSA-P, but not in patients with $\mathrm{PD}[10]$.

This lack of consistency is due to several factors in between-group design (e.g., number of samples, duration of the disease, variability in symptom characteristics, severity of illness, etc.). In light of this limitation, we opted for a withingroup design to explore the effect of progression of the disease on brain atrophy in a group of PD patients.

\section{Methods}

2.1. Subjects. A group of 25 patients with PD were recruited at the Ambulatory of Movement Disorders at the Nuovo Ospedale Sant'Agostino-Estense (Modena). Fifteen healthy volunteers (HV), matched for age and gender, served as controls. 
TABLE 1: Sociodemographic and clinical characteristics of the two study groups. Values denote mean ( \pm standard deviation) or numbers of subjects.

\begin{tabular}{lcc}
\hline & PD patients $N=20$ & Controls $N=15$ \\
\hline Sociodemographics & & \\
Age (years) & $60.5( \pm 7.7)$ & $64.6( \pm 4.8)$ \\
Sex (male/female) & $12 / 8$ & $8 / 7$ \\
Education (years) & $9.75( \pm 3.76)$ & $11.3( \pm 3.19)$ \\
Clinical characteristics & \\
Duration of illness (years) & $5.17( \pm 4.14)$ & \\
Age at diagnosis (years) & $55.20( \pm 8.44)$ \\
Baseline (test) OFF-drug & \\
UPDRS I & $2.50( \pm 1.84)$ \\
UPDRS II & $8.55( \pm 3.88)$ \\
UPDRS III & $26.6( \pm 7.12)$ \\
H\&Y scale & $2.42( \pm 0.25)$ \\
Retest OFF-drug & \\
UPDRS I & $2.61( \pm 1.50)$ \\
UPDRS II & $9.76( \pm 5.21)$ \\
UPDRS III & $22.46( \pm 6.02)$ \\
H\&Y scale & $2.71( \pm 0.26)$ \\
Equivalent dose of & \\
dopaminergic drug & \\
Test (mg) & $473.5( \pm 238.0)$ \\
Retest (mg) & $650.3( \pm 272.9)$ \\
\hline
\end{tabular}

At baseline, all subjects underwent magnetic resonance imaging (MRI) scan, physical examination, and neuropsychological assessment. Those with a poor quality MRI scan or evidence of previous stroke were not included.

In summary, $20 \mathrm{PD}$ patients (mean age $=63 \mathrm{yrs} ; 12$ males and 8 females) and 15 age, gender, and education matched $\mathrm{HV}$ (mean age $=65$ yrs; 8 males and 7 females) were included in the imaging analyses (Table 1).

Patients were diagnosed according to the UK Brain Bank criteria [11]. All patients used dopaminergic medication (levodopa or dopamine agonists). Disease severity was assessed using the Hoehn and Yahr stages and Unified Parkinson's Disease Rating Scale (UPDRS) in OFF-drug condition (at least $18 \mathrm{~h}$ following intake of the last dose of levodopa and $36 \mathrm{~h}$ after other antiparkinsonism medications). Patients were reassessed clinically and MRI after 2 years $($ mean $=25.5 \pm 4.9$ months).

The study was approved by the local ethics committee and written informed consent was obtained from participants prior to the experiment according to the Declaration of Helsinki.

2.2. MRI Acquisition. Scanning was performed at the Department of Neuroscience-Baggiovara, Hospital of Modena University. Three-dimensional (3D) T1-weighted MRI images were acquired using a 3 Tesla Philips Intera MRI scanner. A SPGR pulse sequence (echo time $(\mathrm{TE})=4.6$, repetition time $(\mathrm{TR})=9.9 \mathrm{~ms}$ ) was used. One hundred seventy contiguous sagittal slices were acquired (voxel size $=1 \times 1 \times 1 \mathrm{~mm}$ ) and field of view was $240 \mathrm{~mm}$ with a matrix size of $256 \times 256 \times 170$.

A T2-weighted axial scan and a coronal fluid attenuated inversion recovery (FLAIR) scan were also acquired to have a better definition of possible vascular damage.

2.3. Voxel-Based Morphometry (VBM) Analyses. The optimized VBM protocol was implemented within Matlab 7.1 (Mathworks, Natick, Mass.) through Statistical Parametric Mapping 8 (SPM8 Wellcome Trust Centre for Neuroimaging, London UK).

2.3.1. Preprocessing. Preprocessing was performed using VBM8 (http://dbm.neuro.uni-jena.de/vbm/). This is an improved version of the standard SPM-VBM procedure described in detail elsewhere [12] and already used in previous studies $[12,13]$. In brief, VBM8 comprised several processes: normalization, segmentation, modulation, and smoothing. The normalization step put the individual images in a common space: images were spatially normalized to a widely used T1weighted MRI template in stereotaxic space, the Montreal Neurological Institute/International Consortium for Brain Mapping (MNI/ICBM) 152 standard. In this step, voxel size was resampled to $1.5 \times 1.5 \times 1.5 \mathrm{~mm}$. Then images were segmented in GM, white matter (WM), and cerebrospinal fluid (CSF) volumes. Volumes were then modulated with Jacobian determinants. Modulation involves scaling by the amount of contraction, so that the total amount of GM in the modulated GM volumes remains the same, as it would be in the original images. Finally, segments were smoothed using a $12 \times 12 \times 12$ kernel.

2.3.2. Whole Brain Analysis. Total GM volumes of normalized-modulated images were compared between PD-baseline versus healthy subjects and PD-follow-up versus healthy subjects using a two-sample $t$-test. Since we had to compare the same group (patients at baseline and follow-up; dependent samples) with controls (independent samples), a single ANOVA could not be performed so we decided to evaluate the difference with separate $t$-test for independent samples. For the $t$-test the statistical threshold was set at $P \leq 0.001$ uncorrected for multiple comparison. The extended threshold was differently set at baseline and followup analysis according to SPM approach. For the small volume correction (SVC) the threshold was set at $P<0.05$ with familywise error (FWE) correction [13].

In the $t$-test we used age and TIV (total intracranial volume) as covariates of no interest. In addition, separate correlation analyses were run on the PD data using the following clinical scores: Hoehn and Yahr stages UPDRS scores and disease duration and therapy (levodopa equivalent dose).

The coordinates of significant voxels were converted from MNI space [14] to Talairach and Tournoux coordinates [15] using a Matlab function developed by M. Brett (mni2tal http:// imaging.mrc-cbu.cam.ac.uk/imaging/MniTalairach). Subcortical anatomical regions were identified using the stereotactic atlas of Schaltenbrand-Wahren [16] second edition and the Nieuwenhuys-Voogd-Van Huijzen atlas [17]. 
TABLE 2: Grey matter reduction in PD patients with respect to controls at baseline (test).

\begin{tabular}{lcccc}
\hline & Cluster $k$ & Voxel $Z$ & Level $P$ & MNI coord. $x, y, z$ (mm) \\
\hline R, parietal lobe (BA 40) & 257 & 3.37 & $<0.001$ & $48,-72,45$ \\
R, angular gyrus (BA 39); R, precuneus (BA 31) & & & & $39,-65,32$ \\
R, inferior parietal lobule (BA 7) & & & $<0.05$ & $26,-3,9$ \\
\hline R, putamen* & 57 & 3.77 &
\end{tabular}

$P<0.001$ uncorrected for multiple comparisons (voxel level $k>241$ voxels).

${ }^{*}$ Correction svc $P=0.05$ (FWE).

$\mathrm{L}=$ left and $\mathrm{R}=$ right. $\mathrm{BA}=$ Brodmann area.

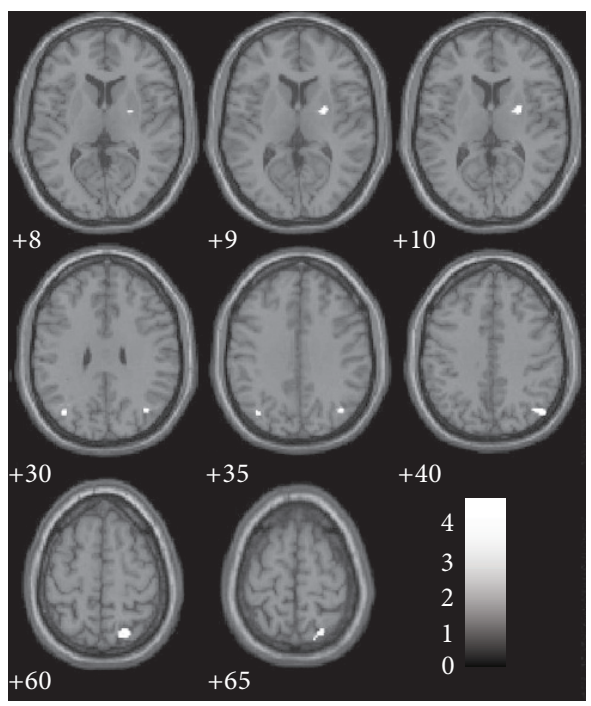

(a)

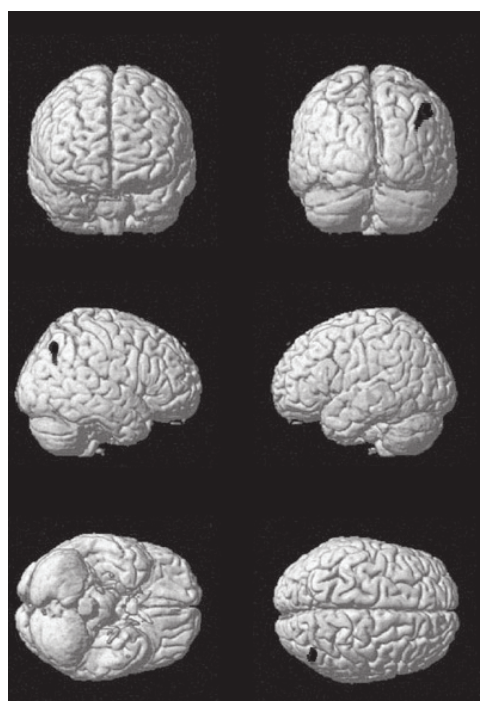

(b)

FIGURE 1: Surface rendering (a) and sagittal sections (b) showing the grey matter reduction in PD patients with respect to controls at baseline (test). Numbers below each sagittal slice represent the $x$ coordinate in MNI space. Clusters are superimposed on the MNI template implemented in SPM8 ( $P=0.001$ uncorrected; $k>241$ voxels $)$.

\section{Results}

3.1. Baseline. We first tested the GM differences between PD patients and HV at baseline. The PD group showed decreased GM volume distributed in the right hemisphere, especially in the precuneus and in the parietal lobe (BA 39, BA 40, and BA 7; Table 2 and Figure 1). Additional GM loss was also identified in right putamen. At a less conservative threshold a symmetrical cluster has been observed also in the left parietal lobe. found.

No significant correlation with clinical parameters was

No significant increased GM volume in PD patients relative to controls was found.

3.2. Follow-Up Study. The comparison between PD patient after two years of follow-up and HV revealed that the PD group showed the same regions of GM loss found at baseline, but wider, involving the right parietal lobe (BA 39, BA 40, BA 7 , and BA 31) and the right putamen (Table 3 and Figure 2). As observed before, a symmetrical not significant decrease of the gray matter was found in the left parietal lobe. Moreover, an additional GM loss cluster was found in the midbrain, in a region identified as the mesencephalic locomotor region (MLR, Figure 2). A similar cluster could be detected at baseline at a less conservative threshold. No significant correlation with UPDRS motor score (considered as a whole, and for specific postural control and path items) was found (UPDRS item $29=1.0 \pm 0.8$ at follow-up; UPDRS item 30 score of $0.9 \pm 0.3$ at baseline and $1.0 \pm 0.4$ at follow-up).

\section{Discussion}

We investigated structural brain differences between groups of healthy subjects and patients with PD using VBM. We evaluated patients at baseline and after two years of disease progression in OFF condition, after a pharmacological washout. The VBM analysis at baseline showed areas of GM loss in the right hemisphere, in particular in the putamen and parietal lobe. These regional atrophies were enlarged after two years of follow-up, where a new cluster in midbrain was also noted.

4.1. Putamen. Structural and functional alterations of the putamen have been widely documented in literature, using different methods. Péran et al. have shown the presence of alterations in the mean diffusivity in the striatum and in 
TABLE 3: Grey matter reduction in PD patients with respect to controls at retest.

\begin{tabular}{lcccc}
\hline & Cluster $k$ & Voxel $Z$ & Level $P$ & MNI coord. $x, y, z$ (mm) \\
\hline $\begin{array}{l}\text { R, parietal lobe (BA 40) } \\
\text { R, angular gyrus (BA 39); R, precuneus (BA 31) }\end{array}$ & 449 & 3.56 & $<0.001$ & $48,-72,46$ \\
$\begin{array}{l}\text { R, inferior parietal lobule (BA 7) } \\
\text { R, superior occipital gyrus (BA 19) }\end{array}$ & & & & \\
\hline R, putamen* & 25 & 3.34 & $<0.001$ & $24,-3,10$ \\
\hline Midbrain* $^{*}$ & 176 & 3.47 & $<0.05$ & $2,-27,-17$ \\
\hline
\end{tabular}

$P<0.001$ uncorrected for multiple comparisons (voxel level $k>241$ voxels).

${ }^{*}$ Correction SVC $P=0.05$ (FWE).

$\mathrm{L}=$ left and $\mathrm{R}=$ right. $\mathrm{BA}=$ Brodmann area.

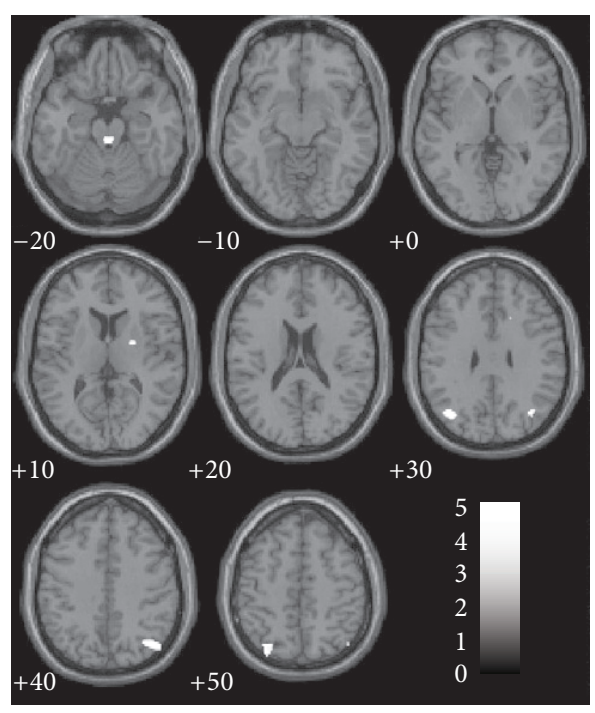

(a)

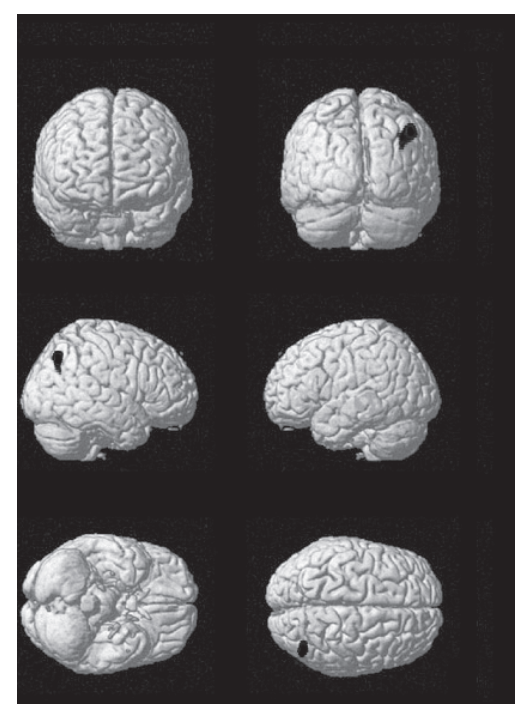

(b)

FIGURE 2: Surface rendering (a) and sagittal sections (b) showing the grey matter reduction in PD patients with respect to controls at retest. Numbers below each sagittal slice represent the $x$ coordinate in MNI space. Clusters are superimposed on the MNI template implemented in SPM8 ( $P=0.001$ uncorrected; $k>223$ voxels).

particular in the right putamen [18] in a group of thirty patients with PD. A dopamine binding transporter loss in this region has been found and neuropathological studies confirmed the presence of microscopic structural alterations such as deposition of Lewy bodies and accumulation of neurofibrillary in the same structures [19]. Molecular studies have recently confirmed the presence of a striatal progressive denervation and atrophy caused by the decrease in dopaminergic afferences due to neuronal loss in the substantia nigra [20].

The GM loss in the putamen in patients at mild to moderate stages of the disease is expected because a significant degeneration of dopaminergic nigrostriatal system precedes the onset of motor symptoms of PD. The right localization did not correlate with the onset side of symptoms nor with the handedness. Although apparently conflicting with data, PD symptoms seem to emerge more often on the dominant hand-side [21]. On the contrary, atrophy does not necessarily depend on nigrostriatal degeneration, which is usually more severe contralaterally to the more affected side. Moreover, it is known that the precentral motor cortex projects to the putamen and to other structures of the basal ganglia of both sides [22]. Therefore, this asymmetry may be related to the complex bilateral connections in the motor system that does not fulfill the side prevalence of clinical signs.

4.2. Parietal Cortex. The atrophy was found in the right parietal regions, in particular in the precuneus (PC) and in the angular gyrus (AG). This GM loss was wider in the follow-up analysis, in agreement with the disease progression. Several studies have shown the presence of atrophy in these parietal areas in PD patients [23, 24], without consistent differences between the two hemispheres. Functional and structural studies emphasized the central role of PC in a wide spectrum of abilities and it participates to the "default mode network" (DMN) a specific, anatomically defined brain system preferentially active when individuals are not focused on the external environment but participate in internal modes of cognition [25].

PC is selectively connected with other parietal structures (parietal caudal operculum and parietal lobules) for the processing of visuospatial information [26-28] and to the premotor cortex $[29,30]$, playing a central role in the visual coordination of hand and reaching movements [31]. 
Subcortical connections of PC are also relevant. They seem to come from the BA 31 region, which is a cortical transition zone from the medial parietal areas to the posterior cingulate and presents an apparent shift in cytoarchitecture from parietal isocortex to limbic cortex [32]. These projections reach several thalamic nuclei, the dorsolateral caudate nucleus, the putamen, the zona incerta, and, mainly, the brainstem structures such as the pretectal area, the superior colliculus, and the nucleus reticularis tegmenti pontis [33]. This last pathway, which brings together fibers coming from IPC, is functionally connected to the retinotectal visual system (RTVS), an ancient and subconscious visual network processing several visual parameters, as quality of oculomotor movements, contrast and colour discrimination, early impaired in PD and strongly involved in the motor behaviour [34].

The AG, particularly on the right side, is involved in higher-order aspects of motor control: the awareness that an intended action is consistent with movement consequences and the awareness of the authorship of the action (called "sense of agency"). Specifically, this region seems to process discrepancies between intended action and movement consequences in such a way that these will be consciously detected by the subject, ability that seems to be compromised in PD patients [35].

The structures of the parietal cortex play a central role in the networks which underlie both sustained attention on current task goals and various forms of response to salient stimuli in the environment to adapt to changing circumstances. In humans, the rostral part seems to be involved in motor planning and action-related functions and it is a part of the human mirror neuron system [36-39]. The right caudal parietal cortex is involved in spatial and nonspatial attention as well as motor preparation $[40,41]$, while left caudal parietal cortex is active during language-related tasks [42, 43]. This hemispheric specialization could explain prevalent atrophy observed in the right side because visuospatial functions, unlike linguistic ones, are often impaired in early PD [44].

Therefore, GM atrophy of the parietal structures observed in our early PD patients can be interpreted as the neuroanatomical correlate of visuospatial symptoms which we know to start early and often during the premotor phase.

4.3. Midbrain. At follow-up, an additional region of decreased grey volume was found in the midbrain. A similar cluster could be detected at baseline at a less conservative threshold. This cluster was located in the posterior region of the midbrain corresponding to the MLR of cat [16]; in humans it includes the pedunculopontine nucleus (PPN), the cuneiform nucleus, the periaqueductal gray, and the locus coeruleus $[45,46]$. It receives inputs from basal ganglia, limbic system and supplementary and premotor cortex and it sends its main efferents to the basal ganglia, the brainstem, and the spinal cord [47].

With respect to the cellular density two main subdivisions of the mammals PPN have been recognized: a pars compacta of PPN (PPNc), constituted by cholinergic neurons; a pars dissipata (PPNd), containing a large proportion of glutaminergic neurons $[48,49]$.
Animal studies revealed a fundamental role of this structure in supraspinal locomotor circuit implicated in control of posture and gait, in particular for the rhythm and fluidity of step. In the decerebrate cat it has been shown [50] that $50 \mathrm{~Hz}$ stimulation of the MLR (corresponding to human PPNd) increased muscle tone and induced alternating hind limb stepping movements, enabling locomotor movements when the treadmill started to move. In the same experiment stimulation of PPN (corresponding to human PPNc) decreased muscle tone.

Anatomical and neurophysiological human studies confirmed such roles of MLR/PPN, in particular in the modulation of posture and gait initiation [47]. In a recent fMRI study on healthy subjects [51], mental imagery of stance and locomotion induced activation of cortical and subcortical areas including cerebellum, PPN, and cuneiform nucleus. This cluster resembled the MLR observed in the cat and demonstrated its similarity with the human locomotor network.

Hathout and Bhidayasiri [52] described three patients with ischemic lesions in the posterior midbrain tegmentum, corresponding to the cluster observed in our study. In addition to ataxia and vertical gaze palsy, probably related to the superior cerebellar pathways (CPS) and to third and fourth nerve impairment, all patients presented a start of hesitation and short and irregular steps without other motor parkinsonian signs.

We know that some axial symptoms in advanced PD, like a beginning hesitation, loss of steps rhythmicity, festination, and freezing, may be not dopa-responder and may depend on the involvement of cholinergic mesencephalic centers. In patients with progressive supranuclear palsy, PD, and Parkinson-Dementia, neuropathologic studies showed a strong loss of cholinergic neurons of PPN, correlating with the severity of disease [47, 53, 54]. Based on these observations some authors choose PPN as target of deep brain stimulation (DBS) in PD improving gait and other axial symptoms $[55,56]$. Studying this model, Ballanger and collaborators showed that unilateral PPN-DBS increased rCBF in different subcortical area interconnected with PPN especially in cerebellum, thalamus, and a brainstem region corresponding to MLR [57].

In a recent VBM-fMRI study Snijders et al. [58] showed the presence, in PD patients with freezing of gait compared with nonfreezers, of an area of atrophy in a small posterior area of the midbrain corresponding to the MLR. Moreover, in the same patients, an fMRI study of motor imagery has shown an area of functional activation in the MLR, probably due to loss of balance between excitatory and inhibitory neurons in attempting to support gait planning and execution [58].

Our study confirmed the presence of GM atrophy in MLR. It is important to underline that, differently to Snijders and collaborators, we observed these data also in mild/ moderate stage of $\mathrm{PD}$, in which no patient had a clinically relevant gait or postural disorder. These data suggest an early impairment of MLR/PPN in PD and its specific role in the pathogenesis of postural and gait disorders.

\section{Conclusions}

The classical theory of the PD evolution developed by Braak did not receive convincing and univocal support by 
the neuroimaging techniques. A recent point of view [59] opposes the fixed temporal ordering of the caudal-cephalic propagation patterns of pathological lesions proposed by Braak et al. This new theory confers a role to the "archaic neural networks" consisting of parts of basal ganglia, midbrain, and parietal regions. An early frailty of this system could produce disrupted automatic gait control, olfactory and visual deficits, impaired emotional face recognition, and REM sleep behavior disorder that are the premotor core of PD. Our data, which show an early atrophy of putamen, parietal cortex and MLR, structures large enough to detect the atrophy, seem to support this hypothesis.

Although the small sample size prevents correcting the results for multiple comparison, our study shows that structural MRI measurement methods could be used to identify the presence of brain atrophy in specific regions known to be associated with $\mathrm{PD}$ pathogenesis, also before the occurrence of clinical symptoms. Furthermore this study has highlighted a progression of atrophy correlated with clinical worsening of patients. We hope that our results will shed light on the neuropathological progression of the PD pathology.

\section{Ethical Approval}

Ethical approval was granted from the local ethics committee and informed consent was obtained from participants.

\section{Conflict of Interests}

The authors declare that there is no conflict of interests regarding the publication of this paper.

\section{Acknowledgment}

The authors thank all study participants and their families, as without their long-term commitment, this study could not have occurred.

\section{References}

[1] H. Braak, K. del Tredici, U. Rüb, R. A. I. de Vos, E. N. H. Jansen Steur, and E. Braak, "Staging of brain pathology related to sporadic Parkinson's disease," Neurobiology of Aging, vol. 24, no. 2, pp. 197-211, 2003.

[2] N. Ibarretxe-Bilbao, E. Tolosa, C. Junque, and M.-J. Marti, "MRI and cognitive impairment in Parkinson's disease," Movement Disorders, vol. 24, supplement 2, pp. S748-S753, 2009.

[3] A. Nagano-Saito, Y. Washimi, Y. Arahata et al., "Cerebral atrophy and its relation to cognitive impairment in Parkinson disease," Neurology, vol. 64, no. 2, pp. 224-229, 2005.

[4] A. Brück, T. Kurki, V. Kaasinen, T. Vahlberg, and J. O. Rinne, "Hippocampal and prefrontal atrophy in patients with early non-demented Parkinson's disease is related to cognitive impairment," Journal of Neurology, Neurosurgery \& Psychiatry, vol. 75, no. 10, pp. 1467-1469, 2004.

[5] E. J. Burton, I. G. McKeith, D. J. Burn, E. D. Williams, and J. T. O'Brien, "Cerebral atrophy in Parkinson's disease with and without dementia: a comparison with Alzheimer's disease, dementia with Lewy bodies and controls," Brain, vol. 127, no. 4, pp. 791-800, 2004.

[6] R. Camicioli, M. M. Moore, A. Kinney, E. Corbridge, K. Glassberg, and J. A. Kaye, "Parkinson's disease is associated with hippocampal atrophy," Movement Disorders, vol. 18, no. 7, pp. 784-790, 2003.

[7] M. P. Laakso, K. Partanen, P. Riekkinen Jr. et al., "Hippocampal volumes in Alzheimer's disease, Parkinson's disease with and without dementia, and in vascular dementia: an MRI study," Neurology, vol. 46, no. 3, pp. 678-681, 1996.

[8] P. Borghammer, K. Østergaard, P. Cumming et al., "A deformation-based morphometry study of patients with earlystage Parkinson's disease," European Journal of Neurology, vol. 17, no. 2, pp. 314-320, 2010.

[9] B. Ramírez-Ruiz, M. J. Martí, E. Tolosa et al., "Longitudinal evaluation of cerebral morphological changes in Parkinson's disease with and without dementia," Journal of Neurology, vol. 252, no. 11, pp. 1345-1352, 2005.

[10] C. Brenneis, K. Egger, C. Scherfler et al., "Progression of brain atrophy in multiple system atrophy: a longitudinal VBM study," Journal of Neurology, vol. 254, no. 2, pp. 191-196, 2007.

[11] A. J. Hughes, S. E. Daniel, L. Kilford, and A. J. Lees, "Accuracy of clinical diagnosis of idiopathic Parkinson's disease: a clinicopathological study of 100 cases," Journal of Neurology Neurosurgery and Psychiatry, vol. 55, no. 3, pp. 181-184, 1992.

[12] C. D. Good, I. S. Johnsrude, J. Ashburner, R. N. A. Henson, K. J. Friston, and R. S. J. Frackowiak, "A voxel-based morphometric study of ageing in 465 normal adult human brains," NeuroImage, vol. 14, no. 1, pp. 21-36, 2001.

[13] K. Mevel, B. Desgranges, J. C. Baron et al., "Which SPM method should be used to extract hippocampal measures in early Alzheimer's disease?" Journal of Neuroimaging, vol. 21, no. 4, pp. 310-316, 2011.

[14] K. J. Friston, J. Ashburner, C. D. Frith, J.-B. Poline, J. D. Heather, and R. S. J. Frackowiak, "Spatial registration and normalization of images," Human Brain Mapping, vol. 3, no. 3, pp. 165-189, 1995.

[15] J. Talairach and P. Tournoux, Co-Planar Stereotaxic Atlas of the Human Brain, Thieme Medical, New York, NY, USA, 1988.

[16] G. Schaltenbrand and W. Wahren, Atlas for Stereotaxy of the Human Brain, Thieme, Stuttgart, Germany, 2nd edition, 1998.

[17] R. Nieuwenhuys, J. Voogd, and C. Van Huijzen, Sistema nervoso centrale-Testo-atlante, Piccin editore, Padova, Italy, 1980.

[18] P. Péran, A. Cherubini, F. Assogna et al., "Magnetic resonance imaging markers of Parkinson's disease nigrostriatal signature," Brain, vol. 133, no. 11, pp. 3423-3433, 2010.

[19] L. D. Perju-Dumbrava, G. G. Kovacs, S. Pirker et al., "Dopamine transporter imaging in autopsy-confirmed Parkinson's disease and multiple system atrophy," Movement Disorders, vol. 27, no. 1, pp. 65-71, 2012.

[20] C. S. Carter and J. W. Dalley, Brain Imaging in Behavioral Neuroscience, Springer, Berlin, Germany, 2012.

[21] S. Yust-Katz, D. Tesler, T. A. Treves, E. Melamed, and R. Djaldetti, "Handedness as a predictor of side of onset of Parkinson's disease," Parkinsonism and Related Disorders, vol. 14, no. 8, pp. 633-635, 2008.

[22] H. Kunzle, "Bilateral projections from precentral motor cortex to the putamen and other parts of the basal ganglia. An autoradiographic study in Macaca fascicularis," Brain Research, vol. 88, no. 2, pp. 195-209, 1975. 
[23] A. Tessitore, M. Amboni, G. Cirillo et al., "Regional gray matter atrophy in patients with Parkinson disease and freezing of gait," American Journal of Neuroradiology, vol. 33, no. 9, pp. 18041809, 2012.

[24] A. M. Meppelink, B. M. de Jong, L. K. Teune, and T. van Laar, "Regional cortical grey matter loss in Parkinson's disease without dementia is independent from visual hallucinations," Movement Disorders, vol. 26, no. 1, pp. 142-147, 2011.

[25] R. L. Buckner, J. R. Andrews-Hanna, and D. L. Schacter, "The brain's default network: anatomy, function, and relevance to disease," Annals of the New York Academy of Sciences, vol. 1124, pp. 1-38, 2008.

[26] L. D. Selemon and P. S. Goldman-Rakic, "Common cortical and subcortical targets of the dorsolateral prefrontal and posterior parietal cortices in the rhesus monkey: evidence for a distributed neural network subserving spatially guided behavior," The Journal of Neuroscience, vol. 8, no. 11, pp. 4049-4068, 1988.

[27] C. Cavada and P. S. Goldman-Rakic, "Posterior parietal cortex in rhesus monkey: II. Evidence for segregated corticocortical networks linking sensory and limbic areas with the frontal lobe," Journal of Comparative Neurology, vol. 287, no. 4, pp. 422-445, 1989.

[28] G. R. Leichnetz, "Connections of the medial posterior parietal cortex (area $7 \mathrm{~m}$ ) in the monkey," The Anatomical Record, vol. 263, no. 2, pp. 215-236, 2001.

[29] P. B. Johnson, S. Ferraina, and R. Caminiti, "Cortical networks for visual reaching," Experimental Brain Research, vol. 97, no. 2, pp. 361-365, 1993.

[30] S. P. Wise, D. Boussaoud, P. B. Johnson, and R. Caminiti, "Premotor and parietal cortex: corticocortical connectivity and combinatorial computations," Annual Review of Neuroscience, vol. 20, pp. 25-42, 1997.

[31] S. Ferraina, "Visual control of hand-reaching movement: activity in parietal area 7m," European Journal of Neuroscience, vol. 9, no. 5, pp. 1090-1095, 1997.

[32] D. N. Pandya and B. Seltzer, "Intrinsic connections and architectonics of posterior parietal cortex in the rhesus monkey," Journal of Comparative Neurology, vol. 204, no. 2, pp. 196-210, 1982.

[33] E. H. Yeterian and D. N. Pandya, "Striatal connections of the parietal association cortices in rhesus monkeys," Journal of Comparative Neurology, vol. 332, no. 2, pp. 175-197, 1993.

[34] M. Tamietto, F. Cauda, L. L. Corazzini et al., "Collicular vision guides nonconscious behavior," Journal of Cognitive Neuroscience, vol. 22, no. 5, pp. 888-902, 2010.

[35] C. Farrer, S. H. Frey, J. D. van Horn et al., "The angular gyrus computes action awareness representations," Cerebral Cortex, vol. 18, no. 2, pp. 254-261, 2008.

[36] M. Iacoboni, "Neural mechanisms of imitation," Current Opinion in Neurobiology, vol. 15, no. 6, pp. 632-637, 2005.

[37] G. Rizzolatti, "The mirror neuron system and its function in humans," Anatomy and Embryology, vol. 210, no. 5-6, pp. 419421, 2005.

[38] C. Keysers and V. Gazzola, "Expanding the mirror: vicarious activity for actions, emotions, and sensations," Current Opinion in Neurobiology, vol. 19, no. 6, pp. 666-671, 2009.

[39] S. Caspers, K. Zilles, A. R. Laird, and S. B. Eickhoff, "ALE meta-analysis of action observation and imitation in the human brain," NeuroImage, vol. 50, no. 3, pp. 1148-1167, 2010.

[40] G. R. Fink, J. C. Marshall, P. H. Weiss, and K. Zilles, "The neural basis of vertical and horizontal line bisection judgments: an fMRI study of normal volunteers," NeuroImage, vol. 14, no. 1, pp. S59-S67, 2001.
[41] M. Corbetta, G. Patel, and G. L. Shulman, "The reorienting system of the human brain: from environment to theory of mind," Neuron, vol. 58, no. 3, pp. 306-324, 2008.

[42] C. J. Price, "The anatomy of language: contributions from functional neuroimaging," Journal of Anatomy, vol. 197, no. 3, pp. 335-359, 2000.

[43] M. Vigneau, V. Beaucousin, P. Y. Hervé et al., "Meta-analyzing left hemisphere language areas: phonology, semantics, and sentence processing," NeuroImage, vol. 30, no. 4, pp. 1414-1432, 2006.

[44] B. E. Levin, M. M. Llabre, S. Reisman et al., "Visuospatial impairment in Parkinson's disease," Neurology, vol. 41, no. 3, pp. 365-369, 1991.

[45] M. L. Shik and G. N. Orlovsky, "Neurophysiology of locomotor automatism," Physiological Reviews, vol. 56, no. 3, pp. 465-501, 1976.

[46] P. J. Whelan, "Control of locomotion in the decerebrate cat," Progress in Neurobiology, vol. 49, no. 5, pp. 481-515, 1996.

[47] P. A. Pahapill and A. M. Lozano, "The pedunculopontine nucleus and Parkinson's disease," Brain, vol. 123, no. 9, pp. 1767$1783,2000$.

[48] M. M. Mesulam, E. J. Mufson, A. I. Levey, and B. H. Wainer, "Cholinergic innervation of cortex by the basal forebrain: cytochemistry and cortical connections of the septal area, diagonal band nuclei, nucleus basalis (substantia innominata), and hypothalamus in the rhesus monkey," Journal of Comparative Neurology, vol. 214, no. 2, pp. 170-197, 1983.

[49] C. Geula, M.-M. Mesulam, and H. Tokuno, "Developmentally transient expression of acetylcholinesterase within cortical pyramidal neurons of the rat brain," Developmental Brain Research, vol. 76, no. 1, pp. 23-31, 1993.

[50] K. Saitoh, T. Isa, and K. Takakusaki, "Nigral GABAergic inhibition upon mesencephalic dopaminergic cell groups in rats," European Journal of Neuroscience, vol. 19, no. 9, pp. 2399-2409, 2004.

[51] K. Jahn, A. Deutschländer, T. Stephan et al., "Imaging human supraspinal locomotor centers in brainstem and cerebellum," NeuroImage, vol. 39, no. 2, pp. 786-792, 2008.

[52] G. M. Hathout and R. Bhidayasiri, "Midbrain ataxia: an introduction to the mesencephalic locomotor region and the pedunculopontine nucleus," American Journal of Roentgenology, vol. 184, no. 3, pp. 953-956, 2005.

[53] R. M. Zweig, W. R. Jankel, J. C. Hedreen, R. Mayeux, and D. L. Price, "The pedunculopontine nucleus in Parkinson's disease," Annals of Neurology, vol. 26, no. 1, pp. 41-46, 1989.

[54] K. Jellinger, "The pedunculopontine nucleus in Parkinson's disease, progressive supranuclear palsy and Alzheimer's disease," Journal of Neurology Neurosurgery and Psychiatry, vol. 51, no. 4, pp. 540-543, 1988.

[55] P. Plaha and S. S. Gill, "Bilateral deep brain stimulation of the pedunculopontine nucleus for Parkinson's disease," NeuroReport, vol. 16, no. 17, pp. 1883-1887, 2005.

[56] A. Stefani, A. M. Lozano, A. Peppe et al., "Bilateral deep brain stimulation of the pedunculopontine and subthalamic nuclei in severe Parkinson's disease," Brain, vol. 130, no. 6, pp. 1596-1607, 2007.

[57] B. Ballanger, A. M. Lozano, E. Moro et al., "Cerebral blood flow changes induced by pedunculopontine nucleus stimulation in patients with advanced Parkinson's disease: a $\left[{ }^{15} \mathrm{O}\right] \mathrm{H}_{2} \mathrm{O}$ PET study," Human Brain Mapping, vol. 30, no. 12, pp. 3901-3909, 2009. 
[58] A. H. Snijders, I. Leunissen, M. Bakker et al., "Gait-related cerebral alterations in patients with Parkinson's disease with freezing of gait," Brain, vol. 134, no. 1, pp. 59-72, 2011.

[59] N. J. Diederich and A. Parent, "Parkinson's disease: acquired frailty of archaic neural networks?" Journal of the Neurological Sciences, vol. 314, no. 1-2, pp. 143-151, 2012. 


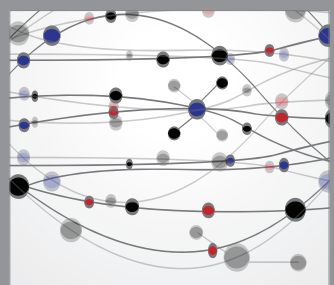

The Scientific World Journal
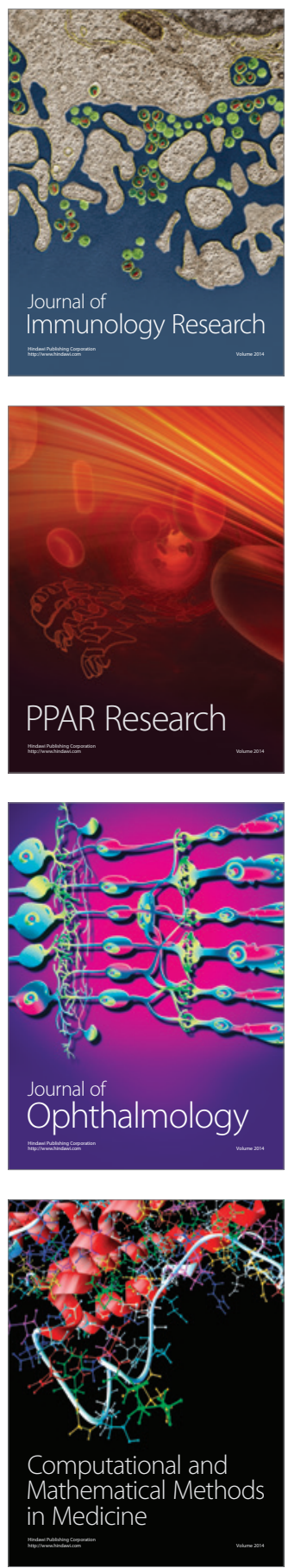

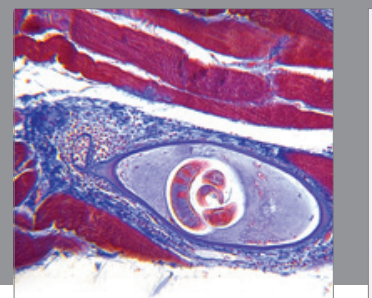

Gastroenterology

Research and Practice
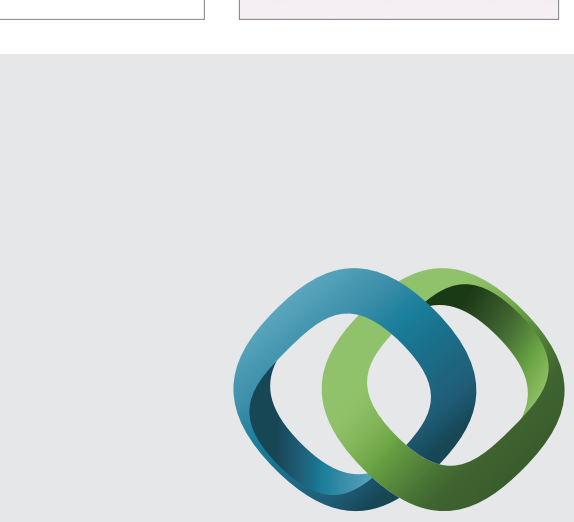

\section{Hindawi}

Submit your manuscripts at

http://www.hindawi.com
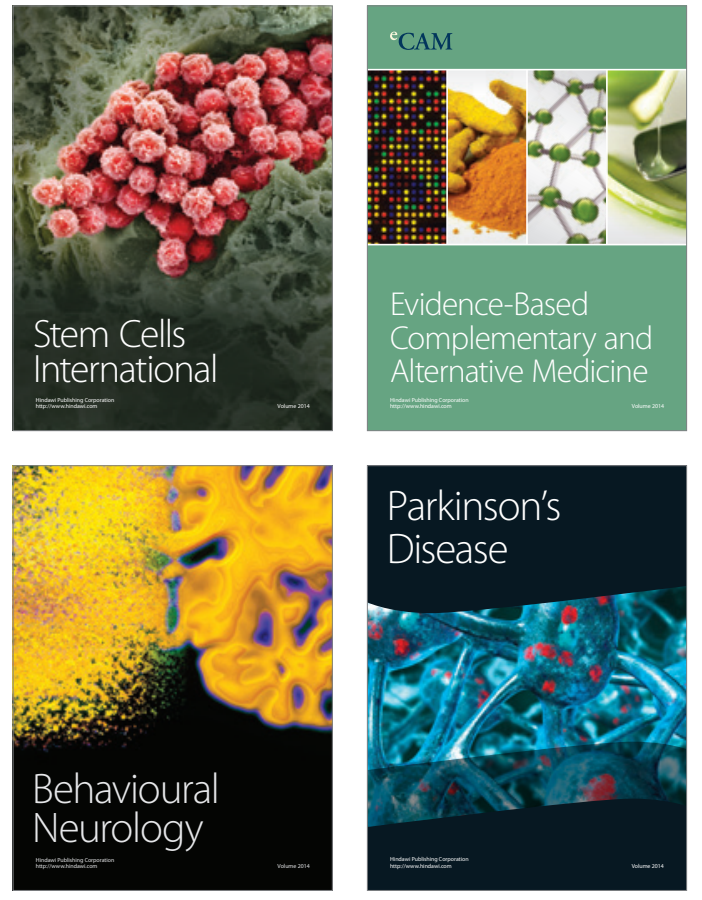
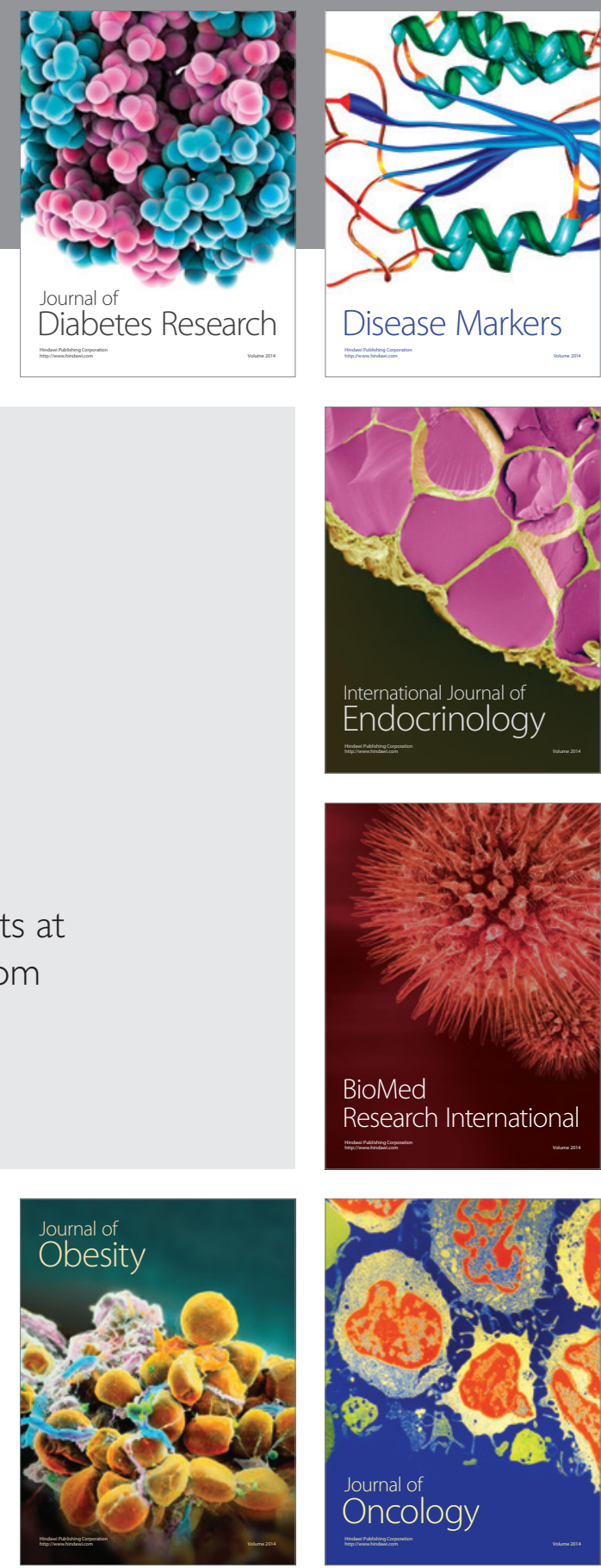

Disease Markers
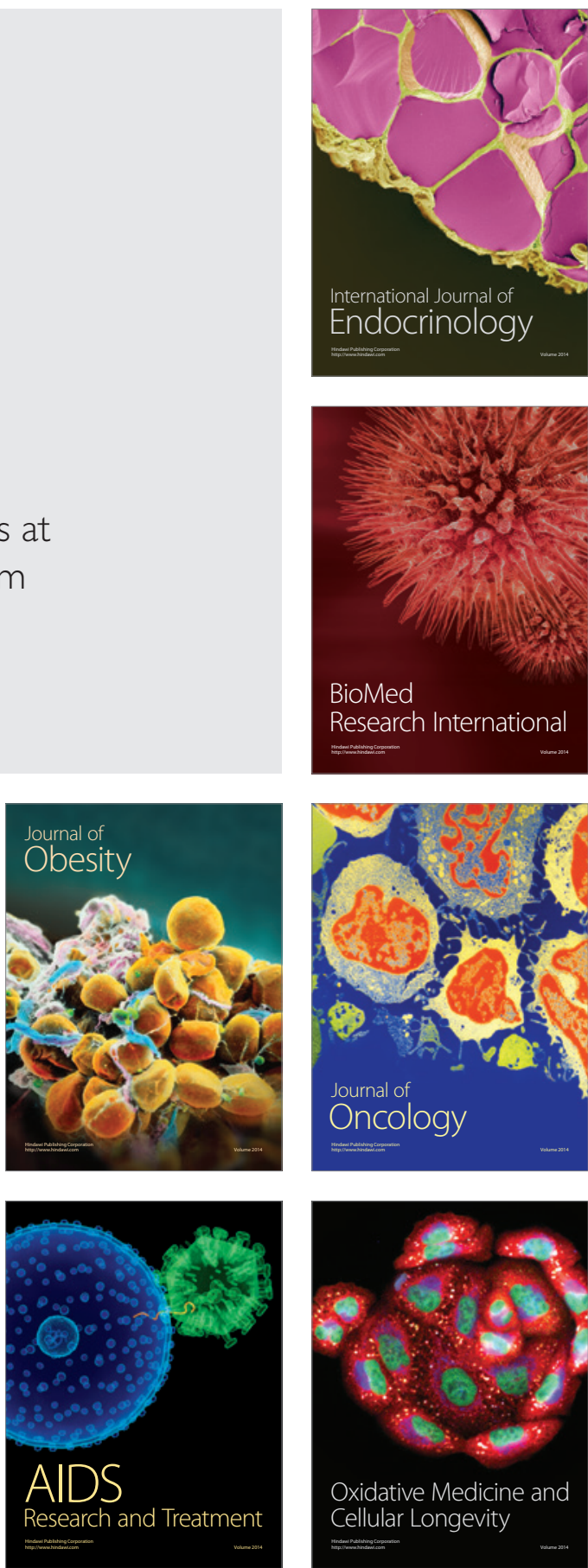